\title{
Adaptive Sliding Mode Control for Magnetic Suspension Short-Stroke Stage in a Reticle Stage
}

\author{
Bizhong $\mathrm{Xia}^{1, \mathrm{a}}$, Shuang $\mathrm{Wu}^{1, \mathrm{~b}}$, Yong Tian ${ }^{1, \mathrm{c}}$, Kaiming Yang $^{2, \mathrm{~d}}$ and $\mathrm{Xin} \mathrm{Li}^{2, \mathrm{e}}$ \\ ${ }^{1}$ Graduate School at Shenzhen, Tsinghua University, Shenzhen, China \\ ${ }^{2}$ Department of Mechanical Engineering, Tsinghua University, Beijing, China \\ axiabz@sz.tsinghua.edu.cn, bwushuang725@126.com, ctian.yong@sz.tsinghua.edu.cn,

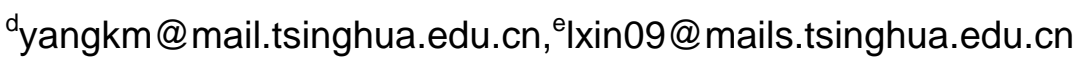
Keywords: external disturbances, adaptive sliding mode control, tracking accuracy, magnetic
suspension short-stroke stage.

\begin{abstract}
The reticle stage in lithographic equipment used to manufacture integrated circuits operates at nanometer accuracy during a scanning process. The modeling errors and external disturbances cause the coupling between multiple degrees of freedom (DOF) of the magnetic suspension short-stroke stage in a reticle stage and as a result, the convergence of the tracking errors cannot be guaranteed. In order to improve the servo performance of the short-stroke stage, this paper presents an adaptive sliding mode control (ASMC) method. The structure of short-stroke stage is introduced, and its dynamic model is established. Based on the established dynamic model, an ASMC is designed. Meanwhile, its convergence and stability are proved by Lyapunov stability theory. The experimental results confirm the effectiveness of the proposed control method compared with the linear feedback control method.
\end{abstract}

\section{Introduction}

In the ultra-precision systems, magnetic suspension positioning stages with voice coil actuators are widely used where ultrahigh precision movement over a micro scale $[1,2]$. However, the application of magnetic drive also introduces some problems. For example, placing multiple actuators close to each other and balance magnets required to compensate the gravity of the stage would result in the electromagnetic crosstalk between these actuators and balance magnets [3]. System uncertainties caused by the machining errors of coil and permanent magnet, thrust fluctuation of actuators and measurement noise would lead to modeling errors. Moreover, the disturbances from the long-stroke stage caused by uneven air gap thickness, cables forces and other disturbances are also coupled to the shot-stroke stage to a certain extent [4]. These disturbances and the modeling errors will heavily affect the positioning accuracy of the stage.

Sliding mode control (SMC) algorithm has been widely used in high-speed and high-accuracy position systems [5, 6], since SMC has good ability to deal with the model uncertainties and external disturbances [7]. SMC drives the system to move along a specific sliding surface and maintain the trajectory on this surface for the subsequent time. Therefore, it is robust and insensitive to modeling errors, system parameters and external disturbances [8]. However, it is difficult to overcome large disturbances for SMC, which can cause the chattering of system. An adaptive control is put forward, which has also been successfully applied in robot manipulators [9]. The main objective of adaptive control is to compensate the uncertainties of the system parameters.

In order to handle problems caused by external disturbances and modeling errors and overcome the SMC's disadvantage, an ASMC method is proposed in this paper. In the system, an adaptive rule with control parameters updated online is applied to reduce the influence of the parameter uncertainties. Then SMC control law with PD control can be used successfully to deal with external disturbances and chattering can be effectively alleviated. The stability and convergence of the proposed controller are proved by Lyapunov stability theory. The tracking performance of the 
proposed controller is experimentally demonstrated on a reticle stage by comparing with the linear control method.

\section{Dynamics Model}

Structure of short-stroke stage in reticle stage. As one of the critical components in lithography machine, the reticle stage consists of a short-stroke stage and a long-stroke stage, shown in Fig. 1. The long-stroke stage, which is floated over the guides by air bearings, consists of two symmetrical sliding blocks with linear actuators and restricted to move in scanning direction, achieving high stiff yet non-contact support and micro-scale positioning precision. The short-stroke stage is suspended on the long-stroke stage by the balance magnets in the core of vertical actuators and driven by eight voice coil actuators, which allow the movement in six degrees of freedom. The actuators coils and balance magnets of the short-stroke stage are fixed on the long-stroke stage, while magnets of the short-stroke actuators are installed on the short-stroke stage. With the structure of dual-stroke stage and magnetic suspension, the reticle stage can achieve nanometer-scale positioning accuracy.

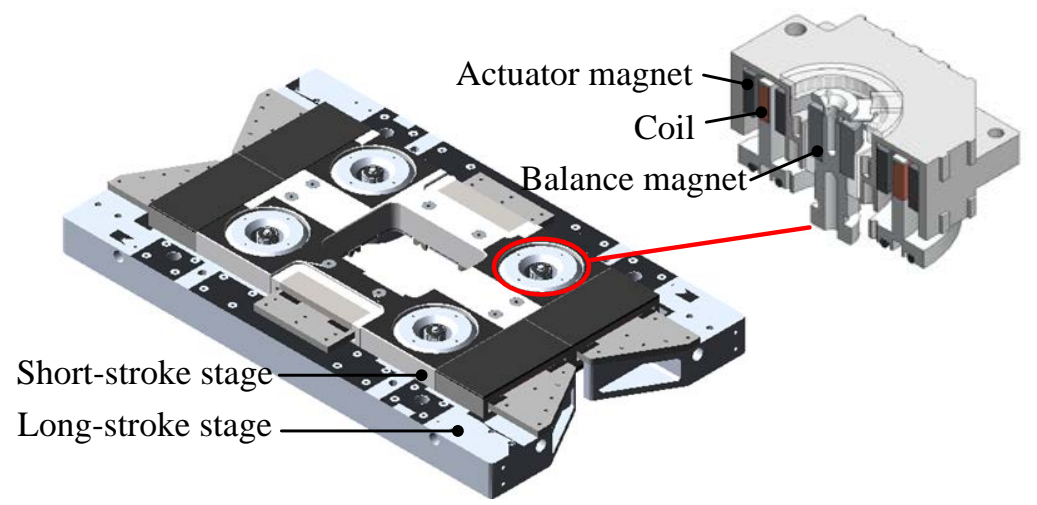

Fig. 1. Structure of the reticle stage

Dynamics model. Generally, the dynamic equation of the short-stroke stage can be written as:

$$
M(q) \ddot{q}+C(q, \dot{q}) \dot{q}+\tau_{d}=\mu
$$

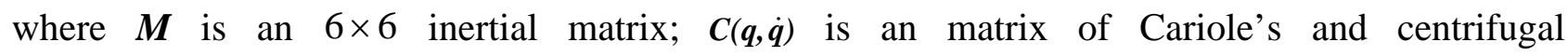
forces, $\boldsymbol{C}(\boldsymbol{q}, \dot{\boldsymbol{q}}) \in \boldsymbol{R}^{6 \times 6} ; \boldsymbol{q}\left(=\left[x, y, \theta_{z}, z, \theta_{x}, \theta_{y}\right]^{T}\right)$ is an $6 \times 1$ vector of position; $; \boldsymbol{u}\left(=\left[F_{x}, F_{y}, T_{z}, F_{z}, T_{x}, T_{y}\right]^{\mathrm{T}}\right)$ is an $6 \times 1$ vector of the control input;; $\tau_{d}$ is the external disturbance.

\section{Adaptive Sliding Mode Control for short-stroke stage}

The Coupling between multiple DOF caused by modeling errors. The control objective is to drive the actual position to the desired position. Define the tracking error as:

$$
\boldsymbol{e}=\boldsymbol{q}-\boldsymbol{q}_{d}=\left(e_{x}, e_{y}, e_{z}, e_{\theta x}, e_{\theta y}, e_{\theta z}\right)^{T}
$$

where $e_{x}, e_{y}, e_{z}, e_{\theta x}, e_{\theta y}, e_{\theta z}$ are tracking errors along the $x, y, z, \theta_{x}, \theta_{y}, \theta_{z}$ axis respectively. The system model cannot be accurately obtained for the presence of external disturbances and the modeling errors, then the linear feedback control law based on inverse dynamics can be written as:

$$
u=\hat{M}(q) \cdot v+\hat{C}(q, \dot{q}) \cdot \dot{q}
$$


where $\hat{\boldsymbol{M}}(\boldsymbol{q})$ and $\hat{\boldsymbol{C}}(\boldsymbol{q}, \dot{\boldsymbol{q}})$ represent nominal model, $\boldsymbol{v}=\ddot{\boldsymbol{q}}_{\boldsymbol{d}}-\boldsymbol{k}_{\boldsymbol{p}} \boldsymbol{e}-\boldsymbol{k}_{d} \dot{\boldsymbol{e}}, \boldsymbol{k}_{\boldsymbol{p}}$ and $\boldsymbol{k}_{\boldsymbol{d}}$ represent controller gains.

With Eq. (3), the difference equation of Eq. (1) can be deduced as:

$$
\begin{aligned}
& \ddot{\boldsymbol{q}}=\boldsymbol{v}-\boldsymbol{\eta}\left(\boldsymbol{q}, \dot{\boldsymbol{q}}, \boldsymbol{v}_{q}\right) \\
& \eta=M^{-1}\left(\tilde{\boldsymbol{M}} \cdot \boldsymbol{v}+\tilde{\boldsymbol{C}} \cdot \dot{\boldsymbol{q}}+\tau_{d}\right)
\end{aligned}
$$

where $\tilde{\mathbf{M}}=\mathbf{M}-\hat{\mathbf{M}}, \tilde{\mathbf{C}}=\mathbf{C}-\hat{\mathbf{C}}$. Eq. (4) indicates that MIMO system cannot be completely decoupled to SISO system for the presence of the uncertainty term. So the error equation of (1) can be written as:

$$
\ddot{e}+k_{d} \dot{e}+k_{p} e=-\eta
$$

(6)

From Eq. (6), it can be seen that the right side of the error equation is not equal to zero. Therefore, the error is not ensured to be convergent in the control of linear controller. If SISO controllers are directly applied to the decoupled system model by feedback linearization, the coupling between multiple degrees of freedom will largely affect the trajectory tracking accuracy of short-stroke stage. In order to reduce the coupling between multiple DOF and improve the robustness of the system, external disturbances and modeling errors must be compensated by the appropriate controller.

Design of ASMC. Define the sliding surface as:

$$
s=\dot{e}+\lambda e
$$

where $\lambda=\operatorname{diag}\left[\lambda_{1}, \lambda_{2}, \lambda_{3}, \lambda_{4}, \lambda_{5}, \lambda_{6}\right], \lambda_{i}>0$. To setup $\boldsymbol{\mu}$, define the reference state as:

$$
\begin{aligned}
& \dot{\boldsymbol{q}}_{r}=\dot{\boldsymbol{q}}-\boldsymbol{s}=\dot{\boldsymbol{q}}_{d}-\lambda \boldsymbol{e} \\
& \ddot{\boldsymbol{q}}_{r}=\ddot{\boldsymbol{q}}-\dot{\boldsymbol{s}}=\ddot{\boldsymbol{q}}_{d}-\lambda \dot{\boldsymbol{e}}
\end{aligned}
$$

From Eq.(8) it can be deduced that:

$$
\begin{aligned}
\boldsymbol{M} \dot{\boldsymbol{s}}+\boldsymbol{C s} & =\boldsymbol{M}\left(\ddot{\boldsymbol{q}}-\ddot{\boldsymbol{q}}_{r}\right)+C\left(\dot{\boldsymbol{q}}-\dot{\boldsymbol{q}}_{r}\right) \\
& =\boldsymbol{u}-\boldsymbol{\tau}_{\boldsymbol{d}}-\left(\boldsymbol{M} \ddot{\boldsymbol{q}}_{r}+C \dot{\boldsymbol{q}}_{r}\right)
\end{aligned}
$$

Choose the control input $\boldsymbol{\mu}$ as:

$$
\begin{aligned}
\boldsymbol{u} & =\hat{\boldsymbol{M}}(\boldsymbol{q}) \cdot \ddot{\boldsymbol{q}}_{r}+\hat{\boldsymbol{C}}(\boldsymbol{q}, \dot{\boldsymbol{q}}) \cdot \dot{\boldsymbol{q}}_{r}-\boldsymbol{k} \cdot \operatorname{sgn}(s)-k_{p} \boldsymbol{e}-\boldsymbol{k}_{d} \dot{\boldsymbol{e}} \\
& =\boldsymbol{Y}\left(\dot{\boldsymbol{q}}_{r}, \ddot{\boldsymbol{q}}_{r}\right) \hat{\boldsymbol{\theta}}-\boldsymbol{k} \cdot \operatorname{sgn}(s)-k_{p} \boldsymbol{e}-k_{d} \dot{\boldsymbol{e}} \\
\dot{\hat{\boldsymbol{\theta}}} & =-\Gamma \boldsymbol{Y}^{T} \boldsymbol{S}
\end{aligned}
$$

where $\boldsymbol{k}=\operatorname{diag}\left[k_{1}, k_{2}, k_{3}, k_{4}, k_{5}, k_{6}\right]$ is a diagonal positive definite matrix. $\boldsymbol{\Gamma}$ is an adaptive gain matrix. Therefore, schematic diagram of the proposed controller can be obtained as Fig. 2. 


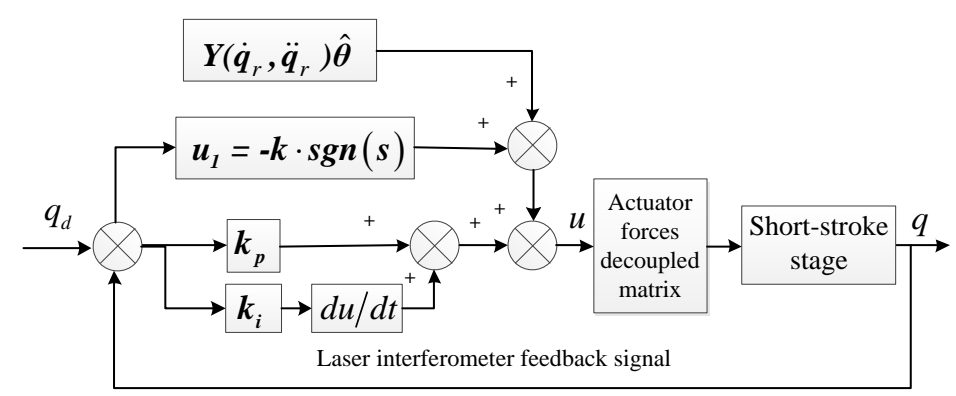

Fig. 2. Schematic diagram of the proposed controller

Stability proof: Based on Eq. (11), Eq. (1) can be rewritten as:

$$
M \dot{s}+C s=-Y\left(\dot{q}_{r}, \ddot{q}_{r}\right) \tilde{\theta}-\boldsymbol{k} \cdot \operatorname{sgn}(s)-k_{p} e-k_{d} \dot{e}-\tau_{d}
$$

where $\tilde{\boldsymbol{M}}=\boldsymbol{M}-\hat{\boldsymbol{M}}, \tilde{\boldsymbol{C}}=\boldsymbol{C}-\hat{\boldsymbol{C}}, \tilde{\boldsymbol{\theta}}=\boldsymbol{\theta}-\hat{\boldsymbol{\theta}}$.

To prove the stability of the system, choose the Lyapunov function as:

$$
V=\frac{1}{2} s^{T} M s+\frac{1}{2} \tilde{\theta}^{T} \Gamma^{-1} \tilde{\theta}+\frac{1}{2} e^{T}\left(k_{p}+\lambda k_{d}\right) e
$$

Since $\dot{\tilde{\boldsymbol{\theta}}}=\dot{\hat{\boldsymbol{\theta}}}$, then it can be derived that:

$$
\begin{aligned}
\dot{\boldsymbol{V}} & =\boldsymbol{s}^{T} \boldsymbol{M \dot { \boldsymbol { s } }}+\frac{\boldsymbol{1}}{\mathbf{2}} \boldsymbol{s}^{T} \dot{\boldsymbol{M}}_{\boldsymbol{s}}+\tilde{\boldsymbol{\theta}}^{T} \boldsymbol{\Gamma}^{-1} \dot{\tilde{\boldsymbol{\theta}}}+\boldsymbol{e}^{T}\left(\boldsymbol{k}_{p}+\lambda \boldsymbol{k}_{d}\right) \dot{\boldsymbol{e}} \\
& =\boldsymbol{s}^{T}\left\{-\boldsymbol{C s}-\boldsymbol{Y}\left(\dot{\boldsymbol{q}}_{r}, \ddot{\boldsymbol{q}}_{r}\right) \tilde{\boldsymbol{\theta}}-\boldsymbol{k} \cdot \boldsymbol{s g n}(\boldsymbol{s})-\boldsymbol{k}_{p} \boldsymbol{e}-\boldsymbol{k}_{d} \dot{\boldsymbol{e}}-\boldsymbol{\tau}_{d}\right\}+\boldsymbol{s}^{T} \boldsymbol{C s}+\tilde{\boldsymbol{\theta}}^{T} \boldsymbol{\Gamma}^{-1} \dot{\hat{\boldsymbol{\theta}}}+\boldsymbol{e}^{T}\left(\boldsymbol{k}_{p}+\lambda \boldsymbol{k}_{d}\right) \dot{\boldsymbol{e}} \\
& =\boldsymbol{s}^{T}\left[-\mathbf{Y}\left(\dot{\boldsymbol{q}}_{r}, \ddot{\boldsymbol{q}}_{r}\right) \tilde{\boldsymbol{\theta}}-\boldsymbol{k} \cdot \boldsymbol{s} \boldsymbol{g n}(\mathbf{s})-\boldsymbol{\tau}_{d}\right]-\lambda \boldsymbol{e}^{T} \boldsymbol{k}_{p} \boldsymbol{e}-\dot{\boldsymbol{e}}^{T} \boldsymbol{k}_{d} \dot{\boldsymbol{e}}+\tilde{\boldsymbol{\theta}}^{T} \boldsymbol{\Gamma}^{-1} \dot{\hat{\boldsymbol{\theta}}} \\
& =\sum_{i=1}^{6}\left\{s_{i}\left[-k_{i} \operatorname{sgn}\left(s_{i}\right)-\tau_{d i}\right]\right\}-\sum_{i=1}^{6} \lambda_{i} k_{p i} e_{i}^{2}-\sum_{i=1}^{6} k_{d i} \dot{e}_{i}^{2}
\end{aligned}
$$

Since $\boldsymbol{M}, \boldsymbol{k}_{\boldsymbol{p}}, \boldsymbol{k}_{\boldsymbol{d}}$ are symmetric and positive definite. Assuming $\left|\tau_{d i}\right|<\left|\tau_{d i}\right|_{\text {bound }}$ has boundary, where $\left|\tau_{d i}\right|_{\text {bound }}$ is the boundary of $\left|\tau_{d i}\right|$, choose $\boldsymbol{k}$ such that $\left|\tau_{d i}\right| \leq k_{i}$, thus

$$
\dot{\boldsymbol{V}}=\sum_{i=1}^{6}\left\{s_{i}\left[-k_{i} \cdot \operatorname{sgn}\left(s_{i}\right)-\tau_{d i i}\right]\right\}-\sum_{i=1}^{6} \lambda_{i} k_{p i} e_{i}^{2}-\sum_{i=1}^{6} k_{d i} \dot{e}_{i}^{2} \leq 0
$$

Thus $\dot{\boldsymbol{V}} \leq 0$. Eq. (15) can be considered as an indicator of the energy of $\boldsymbol{s}$, and it is stable.

\section{Experimental Research}

The proposed method is applied to the short-stroke stage of a reticle stage in lithographic equipment. As shown in Fig. 3, the test bench consists of a motion controller, a data processing unit, D/A converters, motor drivers, short-stroke actuators, long-stroke actuators, a short-stroke stage, a long-stroke stage, measurement systems and A/D converters. The mass of short-stroke stage is $15 \mathrm{~kg}$, the sampling period is $0.2 \mathrm{~ms}$. In this paper, trajectory tracking experiments are foucused on the scanning direction (i.e., $y$ direction) due to the structure limitation and functional demands of the reticle stage as shown in Fig. 1. The target trajectory is shown in Fig. 4. The experiment results using the linear feedback control and the ASMC are shown in Fig. 5 and Table 1. 

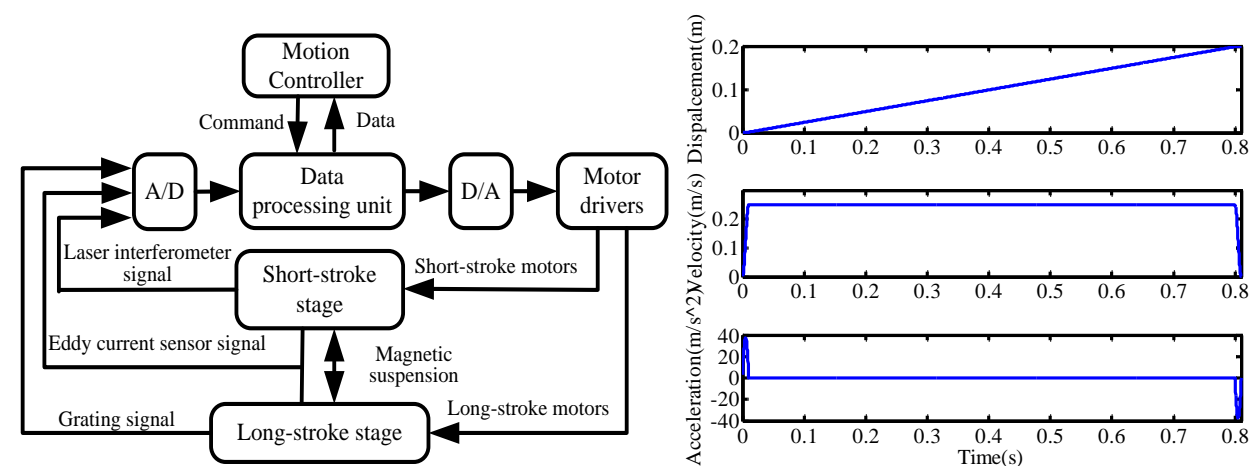

Fig. 3. Schematic diagram of the test bench

Fig. 4 . The target trajectory along $y$ direction
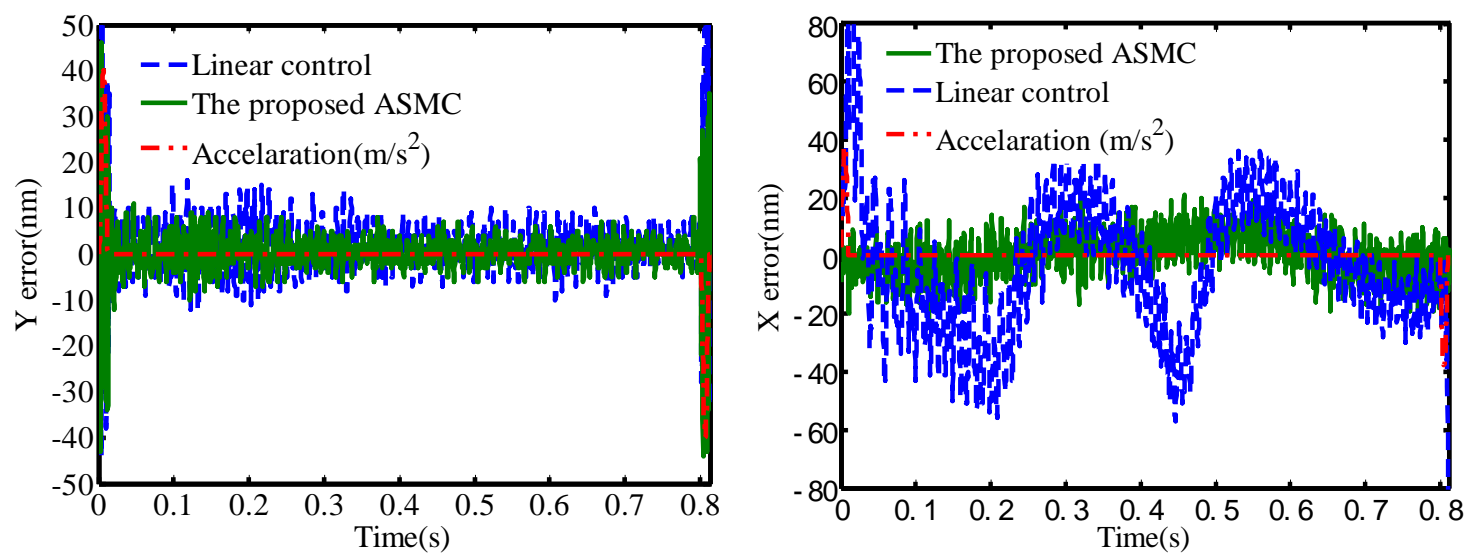

(a). Tracking error in $y$ direction

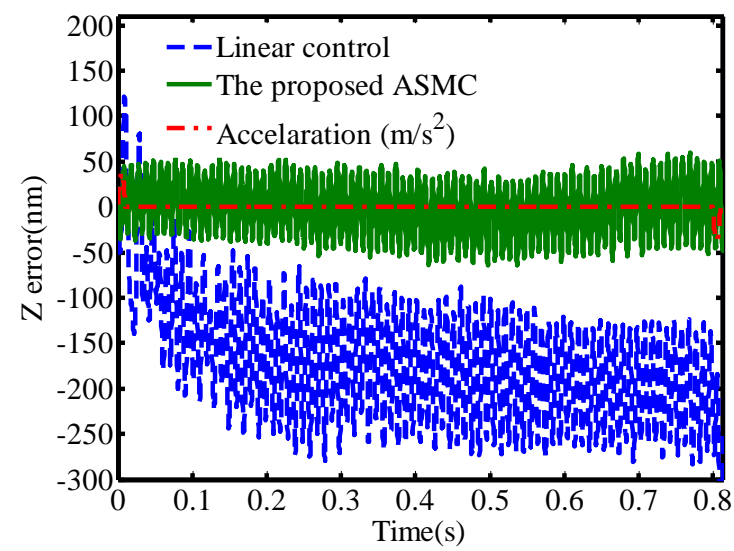

(b). Positioning error in $x$ direction

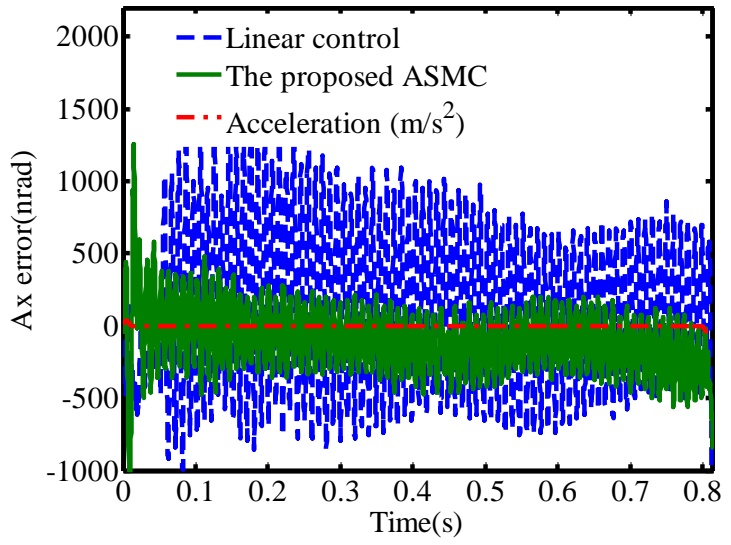

(c). Positioning error in $z$ direction

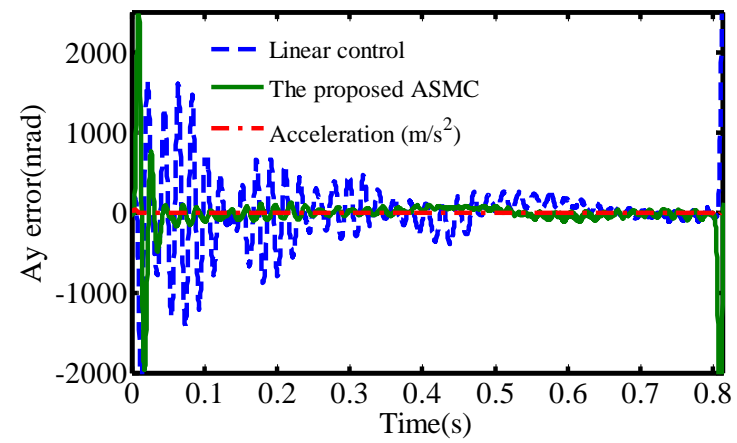

(e). Angular error in $\theta_{y}$ direction

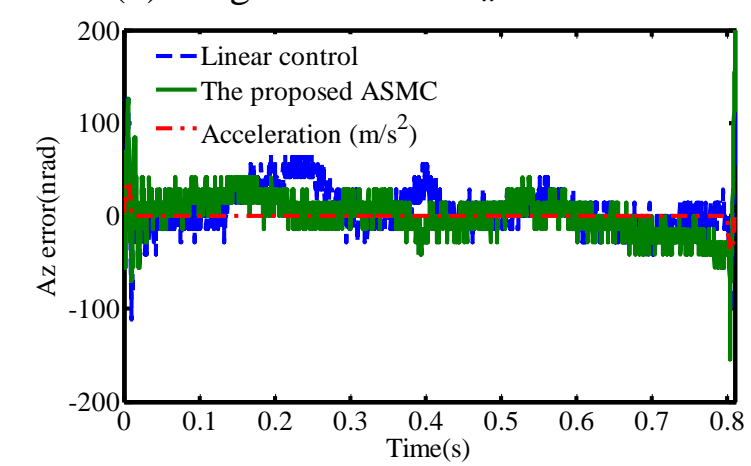

(f). Angular error in $\theta_{z}$ direction

Fig.5. Experimental results with linear feedback control and ASMC 
Table 1. Comparison results of the maximum tracking and positioning errors

\begin{tabular}{ccc}
\hline Maximum errors & \multicolumn{2}{c}{$\begin{array}{c}\text { Lontrol algorithm } \\
\text { Adaptive sliding mode } \\
\text { control }\end{array}$} \\
\hline The tracking error in $y$ direction [nm] & 16 & 11 \\
The positioning error in $x$ direction [nm] & 21 & 43 \\
The positioning error in $z$ direction [nm] & 279 & 48 \\
The angular error in $\theta_{x}$ direction [nrad] & 1455 & 600 \\
The angular error in $\theta_{y}$ direction [nrad] & 1640 & 770 \\
The angular error in $\theta_{z}$ direction [nrad] & 70 & 42 \\
\hline
\end{tabular}

It can be seen from Fig. 5 that the maximum tracking error in constant speed phase is $16 \mathrm{~nm}$ with the linear control algorithm, while the positioning errors of other DOF are also large. This indicates that coupling exists between multiple DOF and the system cannot be completely decoupled just by linear feedback due to the presence of modeling errors and external disturbances. From Table 1, it is concluded that the proposed ASMC effectively reduces the coupling between multiple DOF, the tracking error in scanning direction and the positioning errors in other multiple DOF are also significantly reduced by compensating the modeling errors, external and internal disturbances Therefore, the proposed ASMC can be effectively applied to motion control with system parameter uncertainties and disturbances.

\section{Conclusions}

The ultra-precision motion tracking control of the short-stroke stage is very important for the reticle stage in order to achieve nanometer-scale positioning accuracy. To overcome modeling uncertainties and suppress external disturbances, an ASMC with PD control is proposed. Firstly, the structure of short-stroke stage in reticle stage is introduced and the coupling was analyzed. Secondly, the dynamic model is established and the control approach is proposed. Thirdly, the convergence and the stability of the control law are proved by Lyapunov stability theory. Finally, the control approach was implemented on an experimental reticle stage. The experimental results illustrate that coupling exists between multiple DOF, which affects the accuracy of short-stroke stage in reticle stage. Moreover, the proposed controller effectively reduces the coupling between multiple DOF, the tracking errors in scanning direction and the positioning errors in other multiple DOF by compensating the modeling errors and external disturbances.

\section{References}

[1] de Roover D. Motion control of a wafer stage: A design approach for speeding up ic production[D]. Delft University of Technology, 1997.

[2] Oomen T, van Herpen R, Quist S, et al. Connecting system identification and robust control for next-generation motion control of a wafer stage " IEEE Transactions on Control Systems Technology, 2013, 22(1): 102-118.

[3] Kim W, Trumper D L. High-precision magnetic levitation stage for photolithography. Precision Engineering, 1998, 22(2): 66-77.

[4] Butler H. Position control in lithographic equipment. IEEE Control Systems Magazine, 2011, 31(5): 28-47.

[5] Gutierrez HM and Ro PI. Sliding-mode control of a nonlinear-input system: application to a magnetically levitated fast-tool servo. IEEE Trans Ind Electron 1998; 45: 921-927.

[6] Yang J, Su JY, Li SH, et al. High-order mismatched disturbance compensation for motion 
control systems via a continuous dynamic sliding-mode approach. IEEE Trans Ind Inf 2014; 10: 604-614.

[7] Wheeler G, Su CH and Stepanenko Y. A sliding mode controller with improved adaptation laws for the upper bounds on the norm of uncertainties. Automatica 1998; 34: 1657-1661.

[8] Gutierrez H M, Ro P I. Sliding-mode control of a nonlinear-input system: application to a magnetically levitated fast-tool servo. IEEE Transactions on Industrial Electronics, 1998, 45(6): 921-927.

[9] Slotine J J E, Li W. On the adaptive control of robot manipulators. The International Journal of Robotics Research, 1987, 6(3): 49-59. 